\title{
Pott puffy tumour in a five-year-old girl
}

\author{
Liane Heale MD BPHE, Stacy Zahanova BHSc MD, Zia Bismilla MD MEd
}

A previously healthy five-year-old girl presented to a tertiary care pediatric hospital with a one-month history of frontal headache and decreased appetite. The most recent eight days had been characterized by fever and progressive swelling of the forehead. Three weeks before the presentation, she had been treated with a 10-day course of oral amoxicillin-clavulanic acid for sinusitis. The parents reported no sick contacts or recent travel.

On physical examination, the patient was febrile but fully alert and oriented. Vital signs were otherwise normal. Her forehead was diffusely edematous, and she had bilateral periorbital edema with no conjunctival injection or ocular discharge. There was no evidence of skin abrasion or injury. Her forehead was exquisitely tender to palpation, but the overlying skin was not warm or erythematous. The results of a neurologic examination were normal, with full and painless extraocular movements. An oropharyngeal examination showed normal-sized tonsils without erythema or exudates, and the tympanic membranes were unremarkable.

Computed tomography (CT) of the facial bones, performed earlier at a community hospital, showed diffuse soft-tissue swelling of the forehead and preseptal orbital regions consistent with cellulitis. There was no underlying osteomyelitis, frontal sinusitis or postseptal cellulitis. Blood work showed mild leukocytosis $(12.5 \times$ $10^{9}$ [normal 5-12 $\times 10^{9}$ ] cells/L), with neutrophil count $8 \times 10^{9}\left(\right.$ normal $\left.1.5-8.5 \times 10^{9}\right)$ cells/L, normocytic anemia (hemoglobin 97 [normal 110-140] g/L) and platelet count $504 \times 10^{9}\left(\right.$ normal $\left.150-400 \times 10^{9}\right)$ cells/L. The peripheral blood film was unremarkable. C-reactive protein was elevated, at 133 (normal 0-8) $\mathrm{mg} / \mathrm{L}$. Albumin was 33 (normal 32 to 56) $\mathrm{g} / \mathrm{L}$, and there was no proteinuria. Blood samples were drawn for culture. Given the normal results on the neurologic examination and the extracranial focus of infection, lumbar puncture was not performed. We admitted the patient to the general pediatric service with a presumed diagnosis of periorbital cellulitis and treated her with ceftriaxone and clindamycin, administered intravenously.

With this treatment, the periorbital and forehead swelling resolved, but fevers continued over the next 48 hours as two well-defined masses developed, one on the patient's forehead and one over the crown of her head. Needle aspiration yielded purulent fluid from both masses. Repeat CT showed two subgaleal frontal abscesses with underlying osteomyelitis and intracranial extension into an epidural empyema, which had invaded the superior sagittal sinus (Figure 1). These features confirmed a diagnosis of Pott puffy tumour. The patient was taken directly to the operating room, where the neurosurgical team performed craniectomy and drainage of the subgaleal abscesses. Culture of material from the abscesses revealed the offending bacteria: Streptococcus anginosus. Culture of blood samples drawn at admission showed no growth after five days. The patient recovered fully and completed a 12-week course of intravenous ceftriaxone and metronidazole at home.

\section{Discussion}

Pott puffy tumour is a rare but serious complication of frontal sinusitis most commonly seen in adolescents and young adults. ${ }^{1}$ It is seldom seen in preadolescents. ${ }^{1}$ First described by Percivall Pott in the 18th century, this entity is a subperiosteal abscess overlying osteomyelitis of the frontal bone. It is characterized by localized forehead swelling and most frequently develops after untreated or inadequately treated sinusitis. ${ }^{2,3}$ Although most cases occur in previously healthy children, Pott puffy

\section{KEY POINTS}

- Pott puffy tumour is a rare but important complication of frontal sinusitis that must be considered even in young children.

- The classic presentation includes forehead swelling, headache, rhinorrhea and fever.

- Complete and timely imaging with computed tomography or magnetic resonance imaging is needed to confirm the diagnosis.

- Although intravenous antibiotics are an important initial therapy, the definitive treatment is neurosurgery. 
tumour may also occur as a late complication of neurosurgery, in connection with cocaine abuse or in the context of a dental infection. ${ }^{4}$ It develops when sinusitis spreads to the frontal bone, causing osteomyelitis and extension of purulent material anteriorly or posteriorly. ${ }^{5}$ Adolescents and young

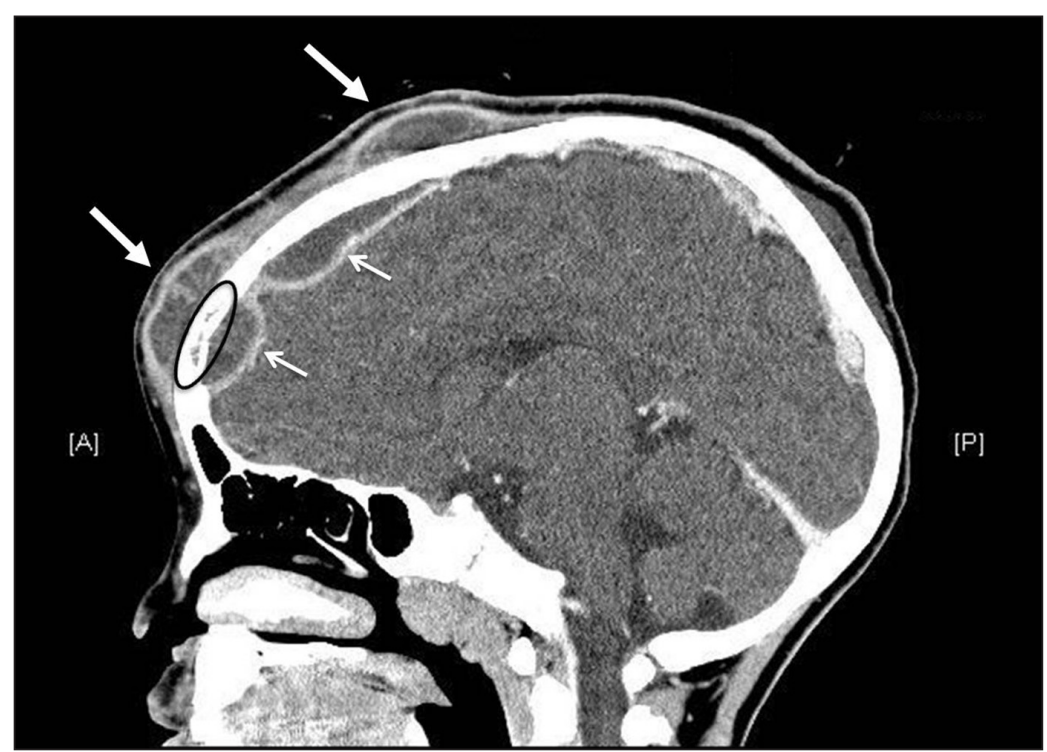

Figure 1: Contrast-enhanced computed tomography of the head of a five-yearold girl with headache and fever showing two large subgaleal abscesses (thick arrows) with osteomyelitis of the frontal bone (circled) and intracranial extension of the infection into an epidural empyema (thin arrows). Note: $A=$ anterior, $\mathrm{P}=$ posterior.

\section{Box 1: Differential diagnosis for forehead swelling in children}

Pott puffy tumour: Subacute development of a well-defined, tender and localized swelling of the forehead associated with headache, rhinorrhea, fever or focal neurologic deficits (rare); ${ }^{4}$ patient may have history of untreated sinusitis or forehead trauma

Sinusitis: Purulent nasal secretions associated with maxillary pain, facial tenderness or tenderness of the teeth lasting at least seven days ${ }^{6}$

Periorbital cellulitis: Eyelid erythema and edema without proptosis or vision changes; may be associated with a history of upper respiratory tract infection, local skin trauma, abscess, insect bite or impetigo ${ }^{9}$

Orbital cellulitis: Eyelid erythema and edema with associated proptosis, decreased visual acuity, and painful or limited extraocular movements; may also be associated with a history of sinusitis, surgery or trauma ${ }^{9}$

Infected sebaceous cyst: Slow-growing and painless mass in hair-bearing skin; may become painful if infected ${ }^{8}$

Carbuncle: Painful swelling of the skin with a palpable fluctuant mass; may be associated with purulent discharge and cellulitis of the surrounding skin ${ }^{8}$

Hematoma: Sudden onset of swelling and ecchymosis of the skin, associated with a history of trauma or injury to the area ${ }^{8}$

Subgaleal hemorrhage: Fluctuant boggy mass developing gradually over the scalp and crossing suture lines; may be associated with coagulation disorders or vascular malformations ${ }^{8}$

Inflammatory cutaneous tuberculosis: Subacute development of subcutaneous swelling, which may progress to ulcerated lesion; should be considered in children with recent travel, immune compromise or other risk factors $^{10}$ adults are believed to be more prone to infection because of a developmental peak in the vascularity of the diploic venous system. ${ }^{1}$ The frontal sinus generally completes development in the preteen years, ${ }^{3,5}$ and Pott puffy tumour therefore tends to be excluded from the differential diagnosis of forehead swelling in younger children; however, as in our case, this may lead to a delay in diagnosis and treatment. In our patient, purulent material collected anterior to the frontal bone in two defined subperiosteal abscesses and tracked posteriorly beyond the frontal bone as an epidural abscess that compressed the frontal lobes.

Canadian guidelines suggest that a 5- to 10-day course of oral amoxicillin (or trimethoprim-sulfamethoxazole for penicillinallergic patients) may be prescribed after a clinical diagnosis of acute bacterial rhinosinusitis, to improve symptom resolution at 14 days. ${ }^{6}$ However, antibiotic therapy is recommended only if the symptoms are considered severe and persist for longer than seven days. Milder cases may be initially treated with a 72-hour trial of intranasal corticosteroids. ${ }^{6}$ This judicious use of antibiotics will ultimately prevent development of antibiotic resistance in common causative organisms (e.g., Streptococcus pneumoniae and Hemophilus influenzae) but may cause an increase in rare complications of sinusitis, such as Pott puffy tumour.

The causative organisms of Pott puffy tumour reflect those seen in acute bacterial rhinosinusitis. ${ }^{4}$ The most commonly cultured organisms include Streptococcus, Staphylococcus, H. influenzae, Enterococcus and anaerobes such as Fusobacterium and Bacteroides. ${ }^{2,4,7}$ Polymicrobial growth has also been reported. ${ }^{4}$ Streptococcus anginosus, which was isolated in this case, has not been described in relation to the condition.

\section{Clinical presentation}

The classic clinical presentation of subperiosteal abscess includes acute or subacute symptoms and signs of forehead swelling, headache, rhinorrhea and fever. ${ }^{4,7}$ The typical description refers to a well-defined, tender and localized swelling of the forehead or scalp overlying the area of frontal osteomyelitis. ${ }^{2,7}$ In some patients, neurologic examination shows abnormalities (e.g., hemiparesis, obtundation and focal neurologic deficits) secondary to the intracranial extension., ${ }^{2,7}$

\section{Differential diagnosis}

The differential diagnosis for forehead swelling in children includes sinusitis, cellulitis, osteomyelitis, carbuncle, infected sebaceous cyst, hematoma and inflammatory cutaneous tubercu$\operatorname{losis}^{8}$ (Box 1). A detailed history and physical 
examination are essential in the assessment of forehead swelling, and Pott puffy tumour should be suspected when there is subacute development of swelling with an underlying history of sinusitis or forehead trauma.

Three-view plain sinus radiography may be used to confirm a clinical diagnosis of sinusitis, and CT is mainly used to assess for potential complications of sinusitis. ${ }^{6}$ If an intracranial complication is suspected, magnetic resonance imaging (MRI) with gadolinium is the imaging modality of choice. ${ }^{7}$ Sensitivity rates for CT and MRI in detecting intracranial abscesses are $92 \%$ and $100 \%$, respectively. ${ }^{7}$ A delay in imaging can delay appropriate treatment and increase the risk of complications, such as epidural and subdural abscesses, meningitis and venous sinus thrombosis. ${ }^{2,4,7}$ As described above, our patient underwent initial timely CT; however, inadequate visualization of the frontal sinus led to the false assumption that Pott puffy tumour had been ruled out.

\section{Management}

Treatment involves both aggressive antibiotic therapy and neurosurgical intervention. ${ }^{7}$ Once the diagnosis is suspected, appropriately targeted intravenous antibiotics with good central nervous system penetration should be initiated after blood samples have been drawn for culture. The goals of neurosurgical treatment include evacuation of the subperiosteal abscess, removal of osteomyelitic bone and removal of granulation tissue. ${ }^{7}$ No optimal surgical approach can be recommended because of limited data from case reports. The most commonly reported procedures include sinus lavage, frontal craniotomy with evacuation of purulent collections and frontal craniectomy. ${ }^{7}$ There have been reports of patients experiencing no improvement after the initial neurosurgical intervention, with a more extensive second operation being required. ${ }^{4}$ After the surgery, patients should complete a sixto eight-week course of antibiotics. ${ }^{1}$ Patients with
Pott puffy tumour generally make a full neurologic recovery following appropriate treatment. ${ }^{1}$

\section{The case revisited}

Following discharge from hospital, our patient recovered well, with no recurrence of her symptoms. She had regular follow-up in the infectious diseases clinic and received a three-month course of oral metronidonazole and amoxicillinclavulanic acid upon completion of her intravenous antibiotic therapy. One year after the initial surgery, she underwent cranioplasty with a polyetheretherketone implant to reconstruct the defect in her frontal bone.

This patient made a full recovery. However, some of her morbidity might have been prevented if the recent history of bacterial sinusitis had been taken into account and the possibility of Pott puffy tumour considered.

\section{References}

1. Forgie SE, Marrie TJ. Pott's puffy tumor. Am J Med 2008;121: 1041-2.

2. Bambakidis NC, Cohen AR. Intracranial complications of frontal sinusitis in children: Pott's puffy tumor revisited. Pediatr Neurosurg 2001;35:82-9.

3. Karaman E, Hacizade Y, Isildak H, et al. Pott's puffy tumor. $J$ Craniofac Surg 2008;19:1694-7.

4. Bhatt YM, Belloso A. Pott's puffy tumor: harbinger of intracranial sepsis. J Neurol Neurosurg Psychiatry 2011;82:547-8

5. Blackman SC, Schleiss MR. Forehead swelling caused by Pott's puffy tumor in a 9-year-old boy with sinusitis. Pediatr Int 2005;47:704-7.

6. Desrosiers M, Evans GA, Keith PK, et al. Canadian clinical practice guidelines for acute and chronic rhinosinusitis. Allergy Asthma Clin Immunol 2011;7:2.

7. Kombogiorgas D, Solanki G. The Pott puffy tumor revisited: neurosurgical implications of this unforgotten entity. J Neurosug 2006;105:143-9

8. O'Neill J. An abscess on the forehead. Am Fam Physician 2007; 75:243-4.

9. Papier A, Tuttle D, Mahar T. Differential diagnosis of the swollen red eyelid. Am Fam Physician 2007;76:1815-24.

10. Sethuraman G, Ramesh V. Cutaneous tuberculosis in children. Pediatr Dermatol 2013;30:7-16.

Affiliations: Department of Paediatrics, The Hospital for Sick Children, Toronto, Ont.

Contributors: All of the authors contributed substantially to conception and design of the work, revised the article critically for important intellectual content, gave final approval of the version to be published and agree to be guarantors of the work. 